\section{Toma de decisiones en hemodiálisis crónica: estudio cualitativo en adultos mayores}

\author{
MARÍA ISABEL CATONI ${ }^{1,2, a}$, SOFÍA P. SALAS ${ }^{1,3}$, \\ EMILIO ROESSLER ${ }^{1,4}$, ANDRÉS VALDIVIESO ${ }^{1,5}$, \\ ANTONIO VUKUSICH ${ }^{1,6}$, M. SOLEDAD RIVERA ${ }^{2, b}$
}

\section{Decision making among older adults in hemodialysis. A qualitative assessment}

Background: In Chile there are 22,310 people in Chronic Hemodialysis (CHD), 53\% of them older adults (OA). Shared decision-making and advance directives $(A D)$ are especially important in $O A$ with end-stage chronic renal failure, since they have greater levels of disability, morbidity and mortality, raising doubts about the benefit of therapy. Aims: To understand the experience in decision making and explore ways to express $A D$, in $O A$ in CHD. Material and Methods: A qualitative phenomenological study, performing 12 in-depth interviews to $O A$ who had been at CHD for at least one year. Results: The analysis revealed four broad comprehensive categories, two related to participation in the decision to enter CHD, namely the experience of subjects as spectators and their lack of interest for decision support and two referred to the expression of $A D$, namely the difficulty in facing their own finitude and resistance to express AD. Conclusions: There is little participation of older adults in the decision about their admission to dialysis therapy, and once they enter the CHD program they are not prepared to discuss $A D$ in general, nor an eventual suspension of dialysis in particular.

(Rev Med Chile 2020; 148: 281-287)

Key words: Advance Care Planning; Aged; Clinical Decision-Making; Kidney Failure, Chronic; Renal Dialysis.
${ }^{1}$ Comité de Ética Sociedad Chilena de Nefrología.

2Escuela de Enfermería, Pontificia

Universidad Católica de Chile. Santiago,

Chile.

${ }^{3}$ Centro de Bioética, Facultad de Medicina,

Clínica Alemana, Universidad del

Desarrollo. Santiago, Chile.

${ }^{4}$ Facultad de Medicina, Clínica Alemana-

Universidad del Desarrollo. Santiago,

Chile.

${ }^{5}$ Facultad de Medicina, Pontificia

Universidad Católica de Chile. Santiago,

Chile.

${ }^{6}$ Facultad de Medicina, Universidad de los

Andes. Santiago, Chile.

aEnfermera Matrona. Magíster en Fundamentación Filosófica, Universidad de los Andes.

'Enfermera. Doctora en Enfermería, Universidad de Sao Paulo.

Fuente de apoyo financiero: Sociedad Chilena de Nefrología (SCHN) proporcionó fondos para la ejecución de la investigación. La SCHN no tuvo influencia en el diseño de la investigación, en la recolección, análisis e interpretación de los datos, ni en la revisión o aprobación del manuscrito.

Recibido el 31 de julio de 2019, aceptado el 11 de febrero de 2020.

Correspondencia a:

María Isabel Catoni Salamanca Vicuña Mackenna 4860. Escuela de Enfermería, Campus San Joaquín, Pontificia Universidad Católica de Chile. Santiago, Chile. mcatoni@uc.cl
L

a enfermedad renal crónica es una causa importante de morbimortalidad mundial, estimándose que más de 1,4 millones de individuos con enfermedad renal terminal (ERT) reciben terapia de sustitución o trasplante renal, con un incremento de $8 \%$ anual, la mayoría de ellos tratados con hemodiálisis crónica (HDC) $)^{1,2}$. En Chile 22.310 personas se encuentran en HDC, siendo $53 \%$ de ellos adultos mayores (AM) ${ }^{3}$.

La toma de decisiones compartida y las voluntades anticipadas (VA) son especialmente importantes en AM, ya que presentan mayor discapacidad, morbilidad y mortalidad, surgiendo dudas con respecto a los beneficios de la terapia $^{4,5}$. En un trabajo anterior demostramos que los principales problemas ético clínicos percibidos por médicos y enfermeras en centros de diálisis (CD) eran: a) la pertinencia de la HDC en pacientes muy deteriorados o con mala calidad de vida, y b) dificultades de comunicación para compartir decisiones o establecer alianzas terapéuticas entre pacientes, familiares y equipo tratante ${ }^{6}$. El presente estudio tiene por objeto comprender la experiencia vivida con respecto 
a la toma de decisiones y explorar las formas de expresar la VA, en AM en HDC.

\section{Metodología}

Se realizó un estudio cualitativo fenomenológico, metodología que permite comprender inductivamente la experiencia personal del fenómeno en estudio. Los supuestos básicos de la fenomenología son: a) la experiencia vivida se muestra a la conciencia de quien porta el fenómeno y se expresa mediante el lenguaje; b) las expresiones de los participantes muestran a la conciencia de los investigadores, la esencia de la experiencia vivida mediante unidades de significado; c) las unidades de significado surgen del análisis de los testimonios realizado por los investigadores ${ }^{7-9}$.

La recolección de información se realizó mediante una entrevista en profundidad a 12 pacientes de dos CD en Santiago de Chile, entre diciembre de 2017 y agosto de 2018. Las entrevistas fueron realizadas por una enfermera entrenada en realizar entrevistas en profundidad y sin relación previa con los pacientes ${ }^{10}$. El número de entrevistas se determinó de acuerdo al criterio de saturación ${ }^{11}$.

Los participantes se seleccionaron de manera intencionada según los siguientes criterios de inclusión: 1) estar en programa de HDC al menos un año, por estimar que en ese momento los pacientes se encuentran más estables y con una mejor perspectiva de la experiencia vivida y sus deseos para el cuidado futuro; 2) tener 65 años o más, pues en esta población suelen aparecer dudas respecto a los beneficios de ingresar a HDC y 3 ) tener capacidad para participar libre y voluntariamente dejando constancia de lo anterior en un Consentimiento Informado (CI). Se excluyeron sujetos que al momento del estudio presentaban diagnóstico de depresión registrado en ficha clínica o carecían de condiciones mentales mínimas para una entrevista, lo que se verificó mediante el test minimental (normal > 14).

Para reclutar a los participantes, la enfermera coordinadora del CD seleccionó a los potenciales pacientes quienes posteriormente fueron contactados por el equipo de investigación y cada participante otorgó su CI. Los participantes fueron seleccionados de manera que estuvieran representados los sexos masculino y femenino, y los diferentes niveles educacionales. De los 17 pacientes contactados, 3 rechazaron participar y 2 no aprobaron el minimental.

La entrevista se realizó en un lugar privado que eligió el participante, tuvo una duración promedio de 51 minutos, y consideró dos grandes ejes temáticos: participación en la decisión de ingresar a HDC y expresión de VA.

El análisis fenomenológico se realizó según Streubert modificado por Rivera ${ }^{12}$. Las entrevistas fueron grabadas y transcritas textualmente. El análisis se realizó de manera secuencial, lo que permitió reorientar la forma de abordar el tema con los pacientes, logrando una mejor aproximación al fenómeno en estudio.

Los procesos de recolección de información y análisis de datos fueron asesorados por una experta en investigación cualitativa fenomenológica. La experta en investigación cualitativa y el investigador principal hicieron un análisis inicial de las entrevistas en conjunto, para posterior discusión con los coautores. Las diferencias se resolvieron por consenso.

Para la credibilidad, se triangularon las categorías y significados con el equipo investigador; la fidelidad se alcanzó fundamentando cada categoría con frases textuales de los participantes; para la auditabilidad se contó con las entrevistas y una matriz de análisis que se completó en forma inductiva, iterativa y emergente; y la transferibilidad de los resultados se consiguió mediante la descripción de las características de los participantes ${ }^{13}$.

El protocolo fue aprobado por el Comité Ético-Científico de la Facultad de Medicina de la Pontificia Universidad Católica de Chile.

\section{Resultados}

Las características de los participantes aparecen en Tabla 1. El análisis reveló cuatro grandes categorías comprensivas, dos relacionadas con la participación en la decisión de ingresar a HDC, y dos referidas a la expresión de las VA.

\section{Participación en la decisión de ingresar a HDC}

Los participantes recuerdan su experiencia de ingreso como la de un espectador, pareciera que no participaron en la toma de decisión. En algunos casos ésta fue tomada por el médico tratante y aceptada por el paciente, en otros el médico la tomó en conjunto con la familia. Los pacientes tie- 
Tabla 1. Características demográficas de los participantes

\begin{tabular}{|lc|}
\hline Variable & \\
\hline Edad: mediana (rango en años) & 74 (66-88) \\
Mujeres: $n$ (\%) & $6(50 \%)$ \\
\hline Tiempo en HDC: mediana (rango en años) & $6(1-27)$ \\
\hline Nivel educacional máximo & \\
$\quad$ Básica & 2 \\
Media & 3 \\
Superior & 7 \\
Estado civil & \\
Casado & 4 \\
Separado & 4 \\
Viudo & 4 \\
Actividad laboral & \\
Sin actividad & 10 \\
Con actividad & 2 \\
\hline Seguro de Salud & \\
FONASA & 6 \\
ISAPRE & \\
Otro & 5 \\
Comorbilidad autoreportada: promedio & 1 \\
(rango) & $2,3(1-4)$ \\
Comorbilidades autoreportadas más & \\
frecuentes & \\
Hipertensión arterial & 3 \\
Diabetes mellitus & \\
Ambas & \\
\hline
\end{tabular}

nen conciencia de esto; sin embargo, no solicitan participar en la decisión; refieren haber confiado en el criterio del médico y en el de su familia en un momento de crisis, como es el diagnóstico de ERT. Asimismo, no reconocen haber recibido información sobre su enfermedad y alternativas terapéuticas, y manifiestan desinterés por mayor apoyo para la toma de decisión. Desde su perspectiva, pareciera que recibieron una indicación médica y no información para decidir. Se percibe temor a contar con más información, reconocen saber lo que vendrá, han visto en otros pacientes el deterioro progresivo y la muerte, y prefieren ignorar qué sucederá en su caso específico (Tabla 2).

Durante su permanencia en HDC los pacientes valoran como relevante la información que reciben de sus pares. Con respecto a incorporarse al programa de trasplante, los participantes han tenido tiempo para informarse y reflexionar al respecto, y son capaces de dar razones sobre esta elección.

\section{Expresión de voluntades anticipadas (VA)}

Se observó dificultad en los participantes para enfrentar su propia finitud. La experiencia de morir y de la muerte se mostró como algo a lo que se teme y de lo cual se evita hablar. Manifestaron temor y rechazo a conversar directamente sobre la muerte, refieren que es un tema incómodo, no conversado en su CD. Es difícil, pues les recuerda la posibilidad de su propia muerte (Tabla 3 ).

Tabla 2. Participación en la decisión de ingresar a HDC

\begin{tabular}{|c|c|c|}
\hline Categorías & Significados & Citas \\
\hline \multirow[t]{2}{*}{$\begin{array}{l}\text { Experiencia del sujeto } \\
\text { como espectador }\end{array}$} & $\begin{array}{l}\text { Decisión tomada por médico/aceptada por el } \\
\text { paciente }\end{array}$ & $\begin{array}{l}\text { "Bueno me dijeron que tenís que dializarte... y } \\
\text { yo en ese sentido si un médico me dice ponte de } \\
\text { cabeza... me pongo de cabeza". E7H }\end{array}$ \\
\hline & $\begin{array}{l}\text { Decisión tomada por médico tratante con la } \\
\text { familia/aceptada por el paciente }\end{array}$ & $\begin{array}{l}\text { "Dijo, vamos, hay que dializar no más y me dia- } \\
\text { lizaron una vez... más conversado con mis hijas } \\
\text { que conmigo en verdad...". E4M }\end{array}$ \\
\hline \multirow[t]{2}{*}{$\begin{array}{l}\text { Carencia y desinterés } \\
\text { por apoyo decisional }\end{array}$} & $\begin{array}{l}\text { Desconocen haber recibido información para la } \\
\text { toma de decisión }\end{array}$ & $\begin{array}{l}\text { "Nada, nada, conmigo no ha conversado nadie } \\
\text { todavía". E2H }\end{array}$ \\
\hline & $\begin{array}{l}\text { No manifiestan necesidad de más información } \\
\text { que la adquirida }\end{array}$ & $\begin{array}{l}\text { "No me interesa... ¿Para que me vas a decir que } \\
\text { de aquí pal 2018... te va a venir el patatun?". } \\
\text { E1H } \\
\text { "No, duda ninguna... con lo que sé... creo que } \\
\text { basta....". E9M }\end{array}$ \\
\hline
\end{tabular}

$\mathrm{E}=$ entrevista; $\mathrm{H}=$ participante hombre; $\mathrm{M}=$ participante mujer. 
Tabla 3. Permanencia en HDC y expresión de las voluntades anticipadas

\begin{tabular}{|c|c|c|}
\hline Categorías & Significados & Citas \\
\hline \multirow[t]{2}{*}{$\begin{array}{l}\text { Dificultad para } \\
\text { enfrentar su propia } \\
\text { finitud }\end{array}$} & $\begin{array}{l}\text { Evita y rechaza hablar sobre su } \\
\text { propia muerte }\end{array}$ & $\begin{array}{l}\text { "Yo jamás pienso en que me voy a morir. Quiero vivir, me siento } \\
\text { joven... y me gustaría no morirme todavía. Hay una parte que } \\
\text { no me gusta de la diálisis... los muertos..., la muerte...". E6H }\end{array}$ \\
\hline & $\begin{array}{l}\text { Asistir a HD es una prioridad, } \\
\text { permite mantener la vida }\end{array}$ & $\begin{array}{l}\text { "Si no sigo (en diálisis) me muero poh... hay que seguir viviendo } \\
\text { mientras pueda..." E12M } \\
\text { "... o sea me ha servido (la diálisis), claro po`o sea imagina si yo } \\
\text { llevo } 7 \text { años ya de vida..." (E6H) }\end{array}$ \\
\hline \multirow[t]{5}{*}{$\begin{array}{l}\text { Resistencia para } \\
\text { expresar VA }\end{array}$} & $\begin{array}{l}\text { Rechazan asumir y conversar } \\
\text { sobre VA, confían en otros }\end{array}$ & $\begin{array}{l}\text { "Bueno, quien va a tomar una decisión de que hacer conmigo, } \\
\text { es mi señora". E1H } \\
\text { "La que más toma la decisión por mí es mi hija mayor". E4M }\end{array}$ \\
\hline & $\begin{array}{l}\text { Perciben claramente cuando hay } \\
\text { que suspender la HD en otros }\end{array}$ & $\begin{array}{l}\text { "Es absurdo traer a diálisis a la persona que está con alzhei- } \\
\text { mer...". E6H } \\
\text { "Es un suplicio enorme... no se lo doy a nadie... he visto a viejos } \\
\text { muy mal... y así los dializan... no... ¿ ipara qué?". E8M }\end{array}$ \\
\hline & $\begin{array}{l}\text { Dos pacientes manifiestan que la } \\
\text { HD no debiera discontinuarse en } \\
\text { ninguna circunstancia }\end{array}$ & $\begin{array}{l}\text { "Hay que hacer todo no más... no hay problema... ya me acos- } \\
\text { tumbré a esto... claro... tengo que vivir así...". E11 H } \\
\text { "Yo los obligaría a dializarse (a pacientes con deterioro cogniti- } \\
\text { vo)..." E7H }\end{array}$ \\
\hline & $\begin{array}{l}\text { Tres pacientes han expresado su } \\
\text { VA a sus familiares }\end{array}$ & $\begin{array}{l}\text { "Yo no quiero que me alarguen la vida artificialmente... Lo digo } \\
\text { cada vez que puedo... Mis hijas... son las que van a tener que } \\
\text { tomar la decisión y están... de acuerdo conmigo". E5M }\end{array}$ \\
\hline & & $\begin{array}{l}\text { "... le tengo dicho a mis hijos que yo no quiero (continuar en } \\
\text { diálisis en caso de deterioro cognitivo)". E8M } \\
\text { "No, yo ya les tengo dicho a todos ya (suspender diálisis en caso } \\
\text { de enfermedad avanzada), que me hagan caso es otra cosa". } \\
\text { E10M }\end{array}$ \\
\hline
\end{tabular}

$V A=$ Voluntades anticipadas; $E=$ entrevista; $H=$ participante hombre; $M=$ participante mujer.

Dado que temen a la muerte, asistir a HD es una prioridad pues comprenden que ello les permite mantenerse vivos, perciben que la inasistencia puede tener consecuencias fatales. Sin embargo, relatan mala adherencia a otros aspectos de la terapia, como dieta y fármacos, los cuales son considerados de menor relevancia para mantener la vida.

Concordante con esta mirada, manifestaron sorpresa, resistencia o desconfianza a la pregunta sobre la posibilidad de expresar su VA para una situación futura en la cual no tuvieran capacidad de tomar decisiones. Cuando se les pregunta quien tomará las decisiones en esa circunstancia, delegan la responsabilidad en personas cercanas como sus hijos, cónyuge o el médico tratante.

Dada la dificultad de los pacientes para hablar directamente sobre su propia muerte, después de la cuarta entrevista se utilizó un camino alternativo: preguntar sobre el caso de otras personas en una condición de salud que podría ameritar suspender la HD (cáncer, daño cognitivo). Ante este escenario hipotético, los entrevistados demostraron capacidad para hablar con mucha propiedad sobre las condiciones de otros pacientes en las cuales ellos estarían de acuerdo con suspender la HD. Contrario a lo anterior, dos pacientes manifiestan que la HD en su caso específico no debiera discontinuarse en ninguna circunstancia, y solo tres refieren que han expresado su voluntad anticipada a sus familiares (Tabla 3 ).

\section{Discusión}

Los resultados sugieren que los AM en HDC tienen escasa participación en la toma de decisión con respecto a ingresar a HD, y que actualmente las VA no parecen ser una alternativa culturalmente relevante en nuestro medio. 
Con respecto a la elección de la terapia sustitutiva, describen un escenario donde el médico diagnostica, indica HDC, y el paciente acepta. Nuestros resultados coinciden con los de múltiples estudios observacionales que muestran que la información recibida es frecuentemente incompleta y tardía para que la persona la discuta con sus familiares o cuidadores $^{5,14-16}$. Los pacientes frecuentemente se sorprenden por un diagnóstico grave y que comprenden con dificultad; por su parte, el médico se centra en cómo resolver la situación para evitar la muerte. El paciente-en ese momento-frecuentemente ignora como la terapia impactará su vida y como progresará su enfermedad ${ }^{5,15}$.

Una revisión sistemática de estudios cualitativos revela que los pacientes perciben imposibilidad real de elegir la terapia, principalmente por tres temas: decisiones tomadas por los médicos, falta de comprensión de alternativas terapéuticas, y limitación de recursos terapéuticos (cupo en CD, disponibilidad de trasplante $\mathrm{u}$ otros $)^{14}$. Los dos primeros coinciden con nuestros hallazgos.

Con respecto al momento en que se entrega la información, al igual que en nuestro estudio, los pacientes relatan sentirse demasiado mal como para asimilarla o que se les exigía una decisión apresurada, sin tiempo para discutir sus opciones con la familia, dada el peligro de morir si no se dializan ${ }^{14-16}$. De hecho, la literatura revela que los médicos explican mejor las alternativas de tratamiento cuando se acerca la necesidad de sustituir la función renal. Esto disminuye el tiempo para tomar decisiones y aumenta las posibilidades de que el paciente se vuelva sintomático o tenga impedimentos cognitivos ${ }^{14}$.

Asimismo, la dependencia para decidir y la aceptación pasiva observadas en nuestro estudio, se describen como estrategias de los pacientes para minimizar emociones negativas como "shock", rabia o miedo, ante el diagnóstico de IRT ${ }^{15}$. Muchos pacientes, incluidos los AM, sienten que si desean vivir tienen que seguir el tratamiento indicado por el médico en que confían; en estricto rigor no hay posibilidad de elección ya que la alternativa es la muerte, lo que es concordante con lo expresado por nuestros entrevistados ${ }^{15,16}$. Para la mayoría de los AM esta aceptación pasiva genera más adelante preguntas sobre el significado y el valor de la terapia, y sobre el valor de sus vidas en $\mathrm{HDC}^{16}$.Los participantes revelan que la información sobre la posibilidad de un trasplante renal se entrega fre- cuentemente después de iniciar $\mathrm{HD}$, al igual que otros estudios ${ }^{14}$. Conviene destacar que los pares son una fuente de información importante, mayor incluso que los clínicos, para tomar decisiones con respecto a las alternativas de tratamiento ${ }^{14}$.

Con relación a las VA, el hallazgo más importante es que los pacientes se resisten a expresarlas y a conversar sobre su propia muerte. Nuestros resultados confirman que la muerte es aún un tema tabú, se evita mencionarla porque se le teme. Este silencio muestra un bloqueo, un no querer saber sobre algo que se admite como una realidad inevitable. Por el contrario, emerge el fuerte deseo de querer vivir y justamente por eso se niega la muerte. El compromiso de los pacientes con la terapia de diálisis revela el valor que otorgan a extender la vida, lo que coincide con los hallazgos de Russ en pacientes de 70 y más años ${ }^{16}$.

La literatura señala que en los CD se trabaja constantemente para mantener la vida y se evita hablar de la muerte; siendo esta una realidad siempre presente ${ }^{16}$. La posibilidad de abandonar el tratamiento para permitir la muerte y la presencia constante de ésta en el CD, son temas pobremente reconocidos y de los que se habla muy poco, tal como relatan nuestros entrevistados ${ }^{16-18}$. Tanto clínicos como pacientes se encuentren fuertemente inmersos en esfuerzos instrumentales, inmediatos, por mantener la vida ${ }^{16}$.

En el caso de presentarse una situación en la cual no tuvieran capacidad de expresar sus deseos, los pacientes refieren confiar en la decisión de personas cercanas como hijos, cónyuge o médico tratante. Lo anterior concuerda con la literatura en el sentido de que a pesar de los esfuerzos legislativos para implementar las VA en países como Estados Unidos de Norteamérica (EE.UU.) y España, la expresión por escrito de estas en pacientes con ERT es bastante baja ${ }^{19,20}$. En EE.UU. alrededor de $30 \%$, siendo la VA más frecuente el deseo de no ser reanimado en caso de paro cardiorrespiratorio ${ }^{21}$; y en España $7,9 \%{ }^{20}$. La poca conciencia sobre la gravedad de la enfermedad se revela en algunos estudios que muestran que la opinión del paciente sobre la retirada de la diálisis no figura habitualmente en las $\mathrm{VA}^{22,23}$.

Contrario a la dificultad que evidenciaron los participantes en el presente estudio para conversar sobre su VA, pacientes españoles en diálisis crónica, al ser consultados directamente sobre sus deseos ante situaciones como coma irreso- 
luble, estado vegetativo, demencia irreversible y enfermedad grave no tratable, son capaces de expresar claramente lo que desean para sí mismos; sin embargo hay que considerar que este último estudio utilizó una encuesta, y puede no ser lo mismo enfrentar una entrevista en profundidad que responder un cuestionario en el que se muestran en forma predeterminada las situaciones futuras a considerar ${ }^{20}$.

Solo tres pacientes en nuestro estudio reconocieron que habían conversado sobre su VA con alguien cercano. En oposición, la mayoría (60,9\%) de los pacientes españoles en diálisis crónica ha conversado su VA con personas cercanas, aunque no para situaciones clínicas específicas lo que dificulta su aplicación ${ }^{20}$. Por otra parte, coincidiendo con la actitud de desconfianza expresada por algunos pacientes en nuestro estudio, $51 \%$ piensa que las VA podrían condicionar la actitud del médico sobre su tratamiento ${ }^{22}$.

En conclusión, los resultados del primer estudio nacional sobre toma de decisiones en AM en HDC muestran que hay escasa participación de los pacientes para decidir su ingreso a terapia dialítica, y que no están preparados para discutir VA en general, ni una eventual suspensión de la diálisis en particular. Dado este escenario, se recomiendan nuevos estudios para comprender mejor cómo viven los chilenos la posibilidad del deterioro y la muerte, cómo contribuir al ejercicio de su autonomía con respecto a la elección de la terapia y el final de la vida, y como desean que se impliquen sus representantes y los clínicos cuando ya no puedan decidir. Independiente de lo anterior, toda sugerencia de protocolo o de acciones a tomar respecto a las VA, debe considerar el temor y rechazo a enfrentar la muerte en nuestra cultura, lo cual sugiere la necesidad de contar con más estudios que profundicen en este aspecto.

Las limitaciones del presente estudio se refieren a que considera solo pacientes con 65 años y más, en los que están sobrerrepresentadas las personas con educación superior con respecto a la media nacional.

\section{Referencias}

1. Ene-Iordache B, Perico N, Bikbov B, Carminati S, Remuzzi A, Perna A, et al. Chronic kidney disease and cardiovascular risk in six regions of the world (ISN-KD-
DC): a cross-sectional study. Lancet Glob Health 2016 May; 4 (5): e307-19.

2. Webster AC, Nagler EV, Morton RL, Masson P. Chronic Kidney Disease. Lancet 2017 Mar 25; 389 (10075): 123852.

3. Poblete H. XXXVIII Cuenta de Hemodiálisis Crónica (HDC) en Chile al 31 de agosto de 2018. Sociedad Chilena de Nefrología, Registro HDC. Disponible en: http// www.nefro.cl/v2/biblio/registro/24.pdf.

4. Eneanya ND, Goff SL, Martínez T, Gutiérrez N, Klingensmith J, Griffith JL, et al. Shared decision-making in end-stage renal disease: a protocol for a multi-center study of a communication intervention to improve endof-life care for dialysis patients. BMC Palliat Care 2015 Jun 12; 14: 30 .

5. Schell JO, Patel UD, Steinhauser KE, Ammarell N, Tulsky JA. Discussions of the kidney disease trajectory by elderly patients and nephrologists: a qualitative study. Am J Kidney Dis 2012 Apr; 59 (4): 495-503.

6. Vukusich A, Catoni MI, Salas SP, Valdivieso A, Browne F, Roessler E. Problemas ético-clínicos en hemodiálisis crónica: percepción de médicos y enfermeras. Rev Med Chile 2016 Jan; 144 (1): 14-21.

7. Streubert H \& Rinaldi D. Phenomenology as a method. In Qualitative Research in Nursing. Philadelphia: Lippincott Williams \& Wilkins. 2011; 5: 72-95.

8. Rivera MS, Herrera LM. Fundamentos fenomenológicos para un cuidado comprensivo de enfermería. Texto Contexto Enferm 2006; 15: 158-63.

9. Grossoehme DH. Research Methodology Overview of Qualitative Research. J Health Care Chaplain 2014; 20 (3): 109-22.

10. Rivera MS, Uribe C, Radünz V. Metodología de entrevistas en investigación cualitativa de enfermería. En: Do Prado ML, De Souza ML, Monticelli M, Cometto MC, Gómez PF, Editores, Investigación cualitativa en enfermería: metodología y didáctica. Serie PALTEX Salud y Sociedad. Número 10. OPS; 2013, Capítulo 14, p 198.

11. Wu YP, Thompson D, Aroian KJ, McQuaid EL, Deatrick JA. Commentary: Writing and evaluating qualitative research reports. J Pediatr Psychol 2016 Jun; 41 (5): 493-505.

12. Vega P, Rivera MS, González R. El transitar del sobrevivir al revivir: comprender la vivencia de padres y madres que han perdido a sus hijos a causa del cáncer. Rev Chil Pediatr 2012; 83 (3): 247-57.

13. Guba E \& Lincoln I. Effective evaluation: improving the Usefulness of evaluation results through responsive and naturalistic approaches. Sn Francisco: Jossey-Bass; 1981.

14. Morton RL, Tong A, Howard K, Snelling P, Webster AC. The views of patients and carers in treatment decision 
making for chronic kidney disease: systematic review and thematic synthesis of qualitative studies. BMJ. 2010 Jan 19; 340: c112.

15. Hussain JA, Flemming K, Murtagh FE, Johnson MJ. Patient and Health Care Professional Decision-Making to Commence and Withdraw from Renal Dialysis: A Systematic Review of Qualitative Research. Clin J Am Soc Nephrol. 2015 Jul 7; 10 (7): 1201-15.

16. Russ AJ, Shim JK, Kaufman SR. The value of "life at any cost": talk about stopping kidney dialysis. Soc Sci Med. 2007 Jun; 64 (11): 2236-47.

17. Goff SL, Eneanya ND, Feinberg R, Germain MJ, Marr L, Berzoff J, Cohen LM, et al. Advance Care Planning: A qualitative study of dialysis patients and families. Clin J Am Soc Nephrol. 2015 Mar 6; 10 (3): 390-400.

18. Davison SN. End-of-life care preferences and needs: perceptions of patients with chronic kidney disease. Clin J Am Soc Nephrol. 2010 Feb; 5 (2): 195-204.

19. Barrio-Cantalejo IMB, Lorda PS \& Gutiérrez JJ. De las voluntades anticipadas o instrucciones previas a la planificación anticipada de las decisiones. Nure Investigación. 2004; 1 (5).

20. Sánchez-Tomero JA, Rodríguez-Jornet A, Balda S, Cigarrán S, Herrero JC, Maduell F, et al. Evaluación de la opinión de los pacientes con enfermedad renal crónica en diálisis respecto al fin de la vida y la planificación anticipada de cuidados. Nefrología 2011; 31 (4): 449-56.

21. United States Renal Data System. 2017 USRDS annual data report: Volume 2: End-Stage Renal Disease. Chapter 12: End-of-life Care for Patients with ESRD: 20002014.

22. Holley JL, Stephen CH, Glover, et al. Failure of advance care planning to elicit patients' preferences for withdrawal from dialysis. Am J Kid Dis 1999; 33: 688-93.

23. Feely MA, Hildebrandt D, Edakkanambeth Varayil J, Mueller PS. Prevalence and Contents of Advance Directives of Patients with ESRD Receiving Dialysis. Clin J Am Soc Nephrol 2016; 11: 2204-9. 